\title{
2010 Scientific Referees
}

(C) The Author(s) 2010. This article is published with open access at Springerlink.com

The quality of the Journal of Orthopaedics and Traumatology depends on the qualified and regular collaboration of renowned scientists who devoted their time to constructively

Roberto Adani (Verona, Italy)

Ezio Adriani (Rome, Italy)

Antonio Andreacchio (Turin, Italy)

Paolo Arrigoni (San Donato Milanese, Italy)

Stefano Artiaco (Turin, Italy)

Claudio Ascani (Rome, Italy)

Giovanni Barbanti Brodano (Bologna, Italy)

Stefano Bastani (Milan, Italy)

Vittorio Bellotti (Barcelona, Spain)

Alexander Berth (Magdeburg, Germany)

Celeste Bertone (San Donato Milanese, Italy)

Kristian Bjorgul (Fredrikstad, Norway)

Andrea Borgo (Vicenza, Italy)

Stefano Boriani (Bologna, Italy)

Elena Maria Brach del Prever (Turin, Italy)

Sergio Brambilla (Milan, Italy)

Massimo Cappella (Modena, Italy)

Marco Cassini (Legnago, Italy)

Maurizio Catagni (Lecco, Italy)

Riccardo Ciatti (Rome, Italy)

Norberto Confalonieri (Milano, Italy)

Bruno Corrado (Naples, Italy)

Landino Cugola (Verona, Italy)

Ferdinando Da Rin (Treviso, Italy)

Maria Cristina D'Agostino (Rozzano, Italy)

Umberto De Bellis (Milan, Italy)

Luigi De Palma (Ancona, Italy)

Marco De Peppo (Rome, Italy)

Shabir A. Dhar (Srinagar, India)

Alberto Di Martino (Rome, Italy)

Ofiram Elisha (Rehovot, Israel)

Pasquale Farsetti (Rome, Italy)

Floria Fischer (Milan, Italy)

Matteo Formica (Genoa, Italy) review the submitted articles. We are indebted to the following experts who reviewed papers which completed the peer-reviewing process within 2010.

Roberto Frairia (Turin, Italy)

Olimpo Galasso (Catanzaro, Italy)

Eduardo Garcia-Rey (Madrid, Spain)

Giorgio Gasparini (Catanzaro, Italy)

Federico Grassi (Novara, Italy)

Tetsuo Hagino (Kofu Yamanashi, Japan)

Massimo Innocenti (Florence, Italy)

Takahiko Kiyama (Fukuoka, Japan)

Elizaveta Kon (Bologna, Italy)

Sujith Konan (London, UK)

Arun Kumar (Cheshire, UK)

Harish V. Kurup (Croydon, UK)

Giovanni Andrea La Maida (Milan, Italy)

Renato Laforgia (Bari, Italy)

Giovanni Lovisetti (Menaggio, Italy)

Giammicola Lucidi (Rimini, Italy)

Giulio Maccauro (Rome, Italy)

Bruno Magnan (Verona, Italy)

Devendra Mahadevan (Leicester, UK)

Pietro Maniscalco (Piacenza, Italy)

Ignazio Marcoccio (Brescia, Italy)

Fabrizio Margheritini (Rome, Italy)

Marcos Almeida Matos (Salvator, Brazil)

Cyril Mauffrey (Birmingham, UK)

Giovanno Merolla (Cattolica, Italy)

Giovanni Monteleone (Rome, Italy)

George Mouzopoulos (Athens, Greece)

Angela Notarnicola (Bari, Italy)

Masahiko Nozawa (Tokyo, Japan)

Matthew E. Oetgen (New Haven, CT, USA)

Antonio Pace (Ancona, Italy)

Roberto Padua (Rome, Italy)

Vinay Parmar (Manchester, UK)

Shelain Patel (London, UK) 
Enrico Pola (Rome, Italy)

Michele Rampoldi (Rome, Italy)

Enrico Rebuzzi (Oderzo, Italy)

Dario Regis (Verona, Italy)

Olav Reikeras (Oslo, Norway)

Giovanni Risitano (Messina, Italy)

Seema Rohilla (Rohtak, India)

Carlo L. Romanò (Milan, Italy)

Claudio Rovesta (Modena, Italy)

Raffaella Russo (Naples, Italy)

Bigham Amin Sadegh (Shahrekord, Iran)

Kabul Ch. Saikia (Assam, India)

Toshinori Sakai (Kuramoto, Japan)

Nicola Santori (Milan, Italy)

Alfredo Schiavone Panni (Campobasso, Italy)

Celeste Scotti (Milan, Italy)

Vincenzo Sessa (Rome, Italy)
Roop Singh (Rohtak, India)

Sutherland A.G. (Aberdeen, UK)

Toshiya Tachibana (Nishinomiya, Japan)

Matthias Therbo (Copenhagen, Denmark)

Pierluigi Tos (Turin, Italy)

Antonio Vadalà (Rome, Italy)

Manish Kumar Varshney (New Delhi, India)

Giovanni Vavalle (Bari, Italy)

Istemi Yucel (Duzce, Turkey)

Luigi Zagra (Milan, Italy)

Giovanni Zanoli (Nonantola, Italy)

Giovanni Zatti (Monza, Italy)

Open Access This article is distributed under the terms of the Creative Commons Attribution Noncommercial License which permits any noncommercial use, distribution, and reproduction in any medium, provided the original author(s) and source are credited. 